\title{
21 Im Vorfeld der Annexion. Das Erstarken des Russländischen Reiches, der „Griechische Plan" und der Vertrag von Küçülk Kaynarca von 1774
}

\author{
Siehst du nicht den Halbmond sinken \\ Trüb in Wolken wetterschwer \\ Mit des Silbers fahlem Blinken \\ Nieder in das schwarze Meer? \\ Als er einstens sturmesschnellen \\ Laufes aus der Wüste stieg, \\ Dunkelten die Dardanellen \\ Und das Lied der Vögel schwieg. [...] \\ Und es tobte um die Wälle \\ Von Byzanz Gewittersturm, \\ Bis der Mond mit blut'ger Helle \\ Still stand auf Sophiens Turm. [... $]^{1}$
}

Der Verfasser dieses vermutlich im zeitlichen Zusammenhang mit dem Krimkrieg (1853-1856) entstandenen Gedichts ist ein offenbar außerhalb von Waldsassen/ Oberpfalz weitgehend vergessener Autor und Altphilologe namens Franz Binhack (1836-1915). ${ }^{2}$ Sein insgesamt 26 Strophen umfassender Reim trägt den Namen „Die orientalische Frage“ und beschreibt deren Ausgangspunkt - nämlich die Einnahme Konstantinopels von 1453 durch die Osmanen - aus Sicht eines gedachten christlichen Europas als Katastrophe, auf welche selbst die Natur mit Schrecken reagierte, denn „das Lied der Vögel schwieg. “ Das Grauen, welches die ChristInnen in Byzanz' erwartete, wird drastisch beschrieben, kennen die einfallenden Muslime doch offenbar kein Pardon mit den Insignien byzantinischer Kultur (die „Griechenkron“ wird von ihnen „zum Klumpen umgeschmolzen“, der Kaiserthron wird „blutbefleckt“") - und schlimmer noch, auch nicht mit den der Christenheit heiligen Dingen:

Am Altare umgestoßen

Hell in Scherben unter Schutt

Lag der Kelch, und ausgeflossen

Trank die Asche Christi Blut.

1 Binhack (2018).

2 Seine lateinische Casus-Lehre ist antiquarisch immer noch zu erwerben: Binhack (1877).

○ OpenAccess. ( 2020 Kerstin S. Jobst, publiziert von De Gruyter. ((c))/BY Dieses Werk ist lizenziert unter der Creative Commons Attribution 4.0 International. https://doi.org/10.1515/9783110520620-023 
Und die Stürme wütend hetzten

Über Land und Meere fort

Auf besudelten, zerfetzten,

Losen Blättern Christi Wort. ${ }^{3}$

Alsdann sei, so der Dichter weiter, über Konstantinopel eine „lange Nacht herein“ gesunken, „[d]reimalhundert Jahr“, ehe sich die Nachtigallen „zum Morgengruß“ gerührt hatten und wieder „Früchte an dem gold'nen Hörn“ gewachsen seien, also in der Bucht des Bosporus in Istanbul, der den europäischen Teil der Stadt in einen südlichen und nördlichen Bereich trennt und wohin zaghaft neues Leben zurückgekehrt sei. ${ }^{4}$ Dreihundert Jahre nach der Eroberung Konstantinopels - in der Mitte des 18. Jahrhunderts - hatten sich die Machtverhältnisse in der Schwarzmeerregion und auch an seinem nördlichen Ufer tatsächlich allmählich zu Ungunsten der Hohen Pforte und damit mittelbar auch des Krim-Chanats verschoben. Dies hatte verschiedene Gründe: Beispielsweise zeigten sich die über so lange Zeit gefürchteten osmanischen und tatarischen Reitertruppen militärtechnisch und strategisch der Armee des Zartums immer weniger gewachsen. Das Militär des Moskauer Zaren setzte nämlich zunehmend auf Artillerie, moderne Schusswaffen sowie Wagenburgen als defensive Formationen gegen die mobile Kavallerie aus dem Süden. Zudem wurden streng disziplinierte kalmückische Söldner verpflichtet, welche in der kriegerischen Auseinandersetzung mit Krimtataren oftmals erfolgreich waren. ${ }^{5}$ Der polnisch-litauische Staat hatte seinerseits die eigene Kavallerie-Taktik gleichsam modernisiert, sodass die berittenen Soldaten nunmehr gemeinsam mit der Infanterie und Artillerie vorgehen und somit dem Ansturm des Gegners besser standhalten konnten, was vor allen Dingen die Nogaier vor Probleme stellte. ${ }^{6}$ Diese Innovationen auf kriegstechnischem Gebiet hatten das Chanat und das Osmanische Reich hingegen weitgehend ,verschlafen'. Hinzu kamen wirtschaftliche Probleme im Inneren: Im Osmanischen Reich hatte sich schon seit dem Ende des 16. Jahrhunderts die Abgabenlast der Bevölkerung drastisch erhöht, Lebensmittel verknappten sich durch den starken Bevölkerungszuwachs im Mittel- und Schwarzmeerraum. Die Gefahr von Aufruhr erhöhte

3 Binhack (2018).

4 Das endgültige „Ende der langen Nacht“, also der osmanischen Dominanz, sah der 1836 geborene Autor rückblickend durch die 1830 erreichte Unabhängigkeit Griechenlands eingeläutet. Der fast ein Jahrzehnt dauernde Unabhängigkeitskampf der Griechen wurde bekanntermaßen von allen europäischen Großmächten einschließlich des Russländischen Reiches unterstützt. Binhack warnte die orthodoxen Griechen aber vor einer Bindung an das Zarenreich, ,jenem Bären, der an deinen Toren harrt“ bzw. „den Adler, der im Schilfe sitzet in dem Donausand.“

5 Vgl. Collins (1975), 274 f.

6 Davies B. (2007), 191. 
sich, was wiederum bewaffnete Kräfte band. ${ }^{7}$ Bei den trotz aller Besonderheiten doch engen Beziehungen zwischen Istanbul und Bağçasaray konnte dies nicht ohne Rückwirkungen auf letzteres bleiben. Beide verabsäumten es zudem, neue wirtschaftliche Strukturen zu implementieren, die den phasenweise schwierigen Sklavenhandel als wichtigen Erwerbszweig hätten ersetzen können. Dieser war, wie ja bereits die Händler in den italienischen Kolonien hatten erfahren müssen (vgl. Kapitel 12) und wie jede andere Ware auch, Konjunkturen unterworfen, sei es aus Gründen der Nachfrage oder einer von außen herbeigeführten Verknappung, die zu Preisanstiegen führte. Die Beschaffung großer Zahlen von Gefangenen war beispielsweise mit dem Erstarken der Kosaken ab Ende des 16. Jahrhunderts (vgl. Kapitel 19) zeitweise schwieriger geworden, stoppten diese doch häufig die tatarischen Raubzüge. ${ }^{8}$ Während des Bündnisses zwischen dem Chanat und den Kosaken unter Het'man Bohdan Chmel'nyc'kyj ab 1648 war es hingegen für die krimtatarische Seite besonders leicht gewesen, Tribute einzutreiben und ,harvesting the steppe for slaves“, so Brian L. Davies. ${ }^{9}$ Nach der kosakisch-russischen Übereinkunft von Perejaslav von 1654 hatten die krimtatarischen Reiterarmeen aber wieder einen schlechteren Zugang zur Steppe, und die Zahl der Gefangenen sank. ,Globale‘ Entwicklungen kamen hinzu, veränderten sich in dieser Phase doch weltweit die Handelsrouten und Warenströme; schließlich wurde der Schwarzmeerhandel zugunsten des transatlantischen nachhaltig geschwächt. Überdies wurden die Akkumulation von Kapital und damit der Aufbau eines funktionierenden Kreditwesens weder in Istanbul noch in Bağçasaray entschieden genug vorangetrieben. Eine Folge daraus war die vergleichsweise geringe Zahl entstehender Manufakturen, die ja ein Merkmal des beginnenden Frühkapitalismus waren. ${ }^{10}$ Und letztlich hatte sich das Moskauer Reich in den ersten Jahrzehnten nach dem Ende der sog. Zeit der Wirren (russ. smuta) um 1613 sowie der Einbindung der Kosaken in das Reichsgefüge deutlich zu stabilisieren vermocht und an Macht gewonnen. ${ }^{11}$

7 Vgl. Matuz (1996), 143-164.

8 Vozgrin (1992), 216-220.

9 Davies B. (2007), 192. Er weist zudem auf den parallelen Aufstieg der Kalmücken als Steppenmacht hin, welche wiederholt in die Gebiete der östlichen Nogaier im Gebiet von Azov eindrangen.

10 Inalcik (1969); Rodinson (1966). Spezielle Untersuchungen zur Wirtschaftsgeschichte des Krim-Chanats fehlen leider.

11 Taagepera (1988). 
Somit kam es nicht von ungefähr, dass aus dieser Zeit die ersten Pläne überliefert sind ${ }^{12}$, dem - aus der Perspektive der nördlichen Nachbarn des KrimChanats - südlichen Anrainer den Garaus zu machen. Interessanterweise wurden konkrete Vorstellungen nicht von polnisch-litauischer bzw. russischer Seite entwickelt, sondern von einem katholischen Kroaten: Juraj Križanić (1618-1683) war Theologe, Schriftsteller und Politiker, und erfuhr u.a. deshalb eine gewisse Berühmtheit, da er der Feldgeistliche des polnischen Königs Jan Sobieski (16291696) während der Zweiten Großen Türkenbelagerung und des Entsatzes von Wien war. Als solcher erlag er einen Tag nach der Schlacht am Kahlenberg seinen sich dabei zugezogenen Verletzungen. ${ }^{13}$ Der sich zwischen 1659 und 1676 im Moskauer Reich aufhaltende Križanić arbeitete zeitweilig im Kreml als Archivar, ehe er beim Zaren in Ungnade fiel und nach Sibirien verbannt wurde. Ob katholische Missionsversuche oder sein Engagement als eine Art Proto-Slavophiler dafür verantwortlich waren, kann hier nicht entschieden werden. In jedem Fall trat er für die Vereinigung aller Slaven unter der Führung des Zartums und (!) des Papstes ein. Voraussetzung für die von Križanić vorgesehene Führungsrolle Moskaus wäre die Konversion der orthodoxen Ostslaven zum lateinischen Ritus gewesen; dies war ein Ansinnen, das beim damaligen Zaren Alexej Michajlovič (1629-1676) nicht auf Zustimmung traf - und der daraufhin offenbar die Verbannung des Kroaten befahl, und dies obgleich der Herrscher den Beinamen „der Sanftmütigste“ (russ. Tišajšij) führte. Križanić skizzierte in seinem zwischen 1663 und 1666 entstandenen Hauptwerk „Politik oder Gespräche über die Herrschaft“ (russ. Politika ili razgovory ob vladatel'stvu) in zwei Kapiteln, wie eine gemeinsame Armee aus Ost-, West- und Südslaven das Chanat würde besiegen können. ${ }^{14}$ Diese Planungen realisierten sich bekanntlich nicht, dennoch: Die Idee war in der Welt, Machtverhältnisse begannen sich zu wandeln, und eine Zäsur in den Beziehungen zwischen dem Zartum und dem Osmanischen Reich und mittelbar auch zum Chanat wurde zumindest in der Rückschau sichtbar.

Eine wichtige Voraussetzung dafür bildete der 1667 ausgehandelte Waffenstillstand von Andrusovo zwischen der Rzeczpospolita und dem Moskauer Staat, der einen mehrjährigen militärischen Konflikt zwischen den beiden Antagonisten beendete. An dessen Folgen lassen sich die Veränderungen der Machtverhältnisse in diesem Teil Europas deutlich erkennen, leitete er doch das Ende der polnischlitauischen Großmachtstellung zugunsten des Zartums ein: Die Rzeczpospolita musste sowohl im Norden Verluste hinnehmen (u.a. den der Woiwodschaft von

12 Nach Fisher (1978), 50, gab es im Umfeld Ivans IV. bereits erste Überlegungen, massiv das Chanat zu bekämpfen. Diese Pläne wurden aber wegen ihrer Aussichtslosigkeit fallen gelassen. 13 Ich folge hier und weiters Heller (1992).

14 Berežkov (1891). 
Smolensk) als auch weiter südlich, ging die Linksufrige Ukraine (östlich des Dneprs) doch genauso verloren wie Kiew und Umgebung; ursprünglich sollte diese auch symbolisch so wichtige Stadt nur für zwei Jahre unter Moskauer Herrschaft gestellt werden, tatsächlich wurde das Gebiet aber dauerhaft Moskau zugeschlagen. Das Kosaken-Het'manat hätte eigentlich als gemeinsame Moskauer-polnisch-litauische Herrschaftssphäre behandelt werden sollen, insofern, als dass es beiden Reichen Kriegsdienst - eben gegen Osmanen und Krimtataren - leisten sollte. Tatsächlich, und dies erwies sich in den nächsten zwei Jahrzehnten als relevant, wurden die Kosaken-Gebiete zu einem Spielball oder, wie es der ukrainischstämmige Historiker Orest Subtelny ausdrückt, „zu einem Objekt“ beider Mächte degradiert. ${ }^{15}$ Auf Dauer bedeutete dieses Abkommen die - auch innere - Spaltung des Het'manats zwischen diesen zwei Herrschaftskonglomeraten. ${ }^{16}$ Polen-Litauen und das Zartum verpflichteten sich in Andrusovo weiters zur gemeinsamen Verteidigung gegen das Osmanische Reich und das Krim-Chanat. Für den russischen Historiker Vozgrin markiert diese Übereinkunft zugleich eine Wende in der russischen außenpolitischen Kommunikation, wurde hier doch erstmalig bewusst eine dezidiert antimuslimische Propaganda bemüht. ${ }^{17}$

Im ersten sog. Russisch-Osmanischen Krieg von 1676 bis 1681 - insgesamt gab es bis 1877-1878 zehn - wurde die ganze Komplexität der nördlichen Schwarzmeerregion mit allen ihren Akteuren noch einmal deutlich ${ }^{18}$ : Das Osmanische Reich stritt gemeinsam mit den Krimtataren gegen das Zartum um die Gebiete, die heute zur südlichen Ukraine gehören. Gleichzeitig kämpften verschiedene Kosaken-Führer auf beiden Seiten und gegeneinander. Am bekanntesten wurde Petro Dorošenko (1627-1698), Het'man der Rechtsufrigen Ukraine. Dieser verbündete sich mit den ,ungläubigen“- aus der Perspektive der Orthodoxie - Krimtataren und Osmanen gegen das von den Kosaken der Linksufrigen Ukraine unterstützte Moskauer Reich - und verlor. ${ }^{19}$ Dieser Waffengang endete schließlich 1681 mit dem Vertragswerk von Bağçasaray, das einen zwanzigjährigen Waffenstillstand begründen sollte (was es nicht tat, wie sich zeigen sollte) und die osmanischrussische Grenze entlang des Dneprs festlegte. Weiter wurden Ansiedlungsverbote für alle Beteiligten zwischen dem Südlichen Bug (poln. Boh; ukr. Pivdennyj Buh;

15 Subtelny (1993), 96.

16 Hoensch (1983), 150. Letztlich hatte sich die Aufspaltung der Kosaken schon seit 1654 abgezeichnet, was in der ukrainischen Historiographie zumeist als schmerzliche Spaltung des protonationalen ukrainischen ,Staates“ angesehen und als „Rü̈n““ (ukr. für Ruin) bezeichnet wird. Vgl. Jobst (2015b), 111.

17 Vozgrin (1992), 226.

18 Zum Verlauf insgesamt vgl. Davies B. (2007), 159-172.

19 Hierzu im Detail Subtelny (1993), 96-98. 
russ. Južnyj Bug) und dem Dnepr vereinbart, womit eine Art Pufferzone entstehen sollte. ${ }^{20}$ Für die Geschichte der Krim, die ja im Mittelpunkt des Interesses steht, ist Folgendes an diesem Friedensschluss von Relevanz: Zum einen wurde den Nogaiern trotz der erwähnten Ansiedlungsbeschränkungen weiterhin das Recht zugestanden, durch diese Gebiete zu ziehen, was ihre Lebensgrundlage sichern sollte. Zum anderen führte Chan Murad Giray (1627-1696) die Verhandlungen in Bağçasaray als Stellvertreter des Sultans, was abermals auf die wichtige Position des Chanats im Gefüge des Osmanischen Imperiums hinweist. Allerdings, so zumindest der habsburgische Orientalist Hammer-Purgstall, gingen die Chane fortan dieser Funktion verlustig:

Bis jetzt hatte unmittelbarer Verkehr von Gesandten zwischen Russland und der Krim stattgefunden. Weil Murad Geraj aber die russischen Gesandten misshandelte, wurden von nun an keine russischen Gesandten mehr nach der Krim gesandt und der diplomatische Verkehr Russlands blieb auf Konstantinopel [=Istanbul] beschränkt. ${ }^{21}$

Welche Misshandlungen an russischen Gesandten Murad Giray, der vor allen Dingen wegen seiner Teilnahme und Vorbehalte gegenüber der sog. Großen Türkenbelagerung von 1683 bekannt wurde, begehen ließ, ist nicht bekannt; der Bedeutungsverlust des Chanats wurde in jedem Fall sichtbar. ${ }^{22}$

Die territoriale Integrität des Chanats - sofern im Kontext einer im Norden ja eher offenen, fluiden Grenze überhaupt davon die Rede sein kann - wurde von diesen Ereignissen nicht berührt. ${ }^{23}$ Erste konkrete Versuche zur Beendigung der Existenz dieses Gemeinwesens durch das Zartum folgten aber bereits wenige Jahre später, durch die russischen Kampagnen der Jahre 1687 und 1689 unter dem Kommando von Vasilij V. Golicyn (1643-1714). Durch den ein Jahr zuvor erfolgten Beitritt des Moskauer Reiches zur antiosmanischen Heiligen Liga (ursprünglich einem Bündnis des Heiligen Römischen Reiches Deutscher Nation, des Papsttums, Polen-Litauens und Venedigs) konnte das Moskauer Reich das Vordringen

20 Vgl. genauer Davies B. (2007), $171 \mathrm{f}$.

21 Hammer-Purgstall (1970), 171. Es ist bemerkenswert, mit welcher Hartnäckigkeit christliche AutorInnen an der anachronistischen Bezeichnung „Konstantinopel“ für „Istanbul“ festhielten. 22 Zur Herrschaft des zwischen 1678 und 1683 regierenden Chans, der auf Bitten der Clans vom Sultan wieder abgesetzt wurde, vgl. Hammer-Purgstall (1970), 169-171. Es steht zu vermuten, dass Murad Giray auch deshalb abgesetzt wurde, da er das Scharia-Gericht, das bis dahin einem von den geistlichen Autoritäten in Istanbul eingesetzten Mufti unterstand, zugunsten eines dem Chan verpflichteten Hofgerichtes schwächte. Diese Stärkung der Position des Chans dürfte weder den Mirza noch dem Sultan gefallen haben.

23 Ohnehin verzichtete das Chanat gegenüber seinen nördlichen Nachbarn wohl schon aus Kostengründen auf eine konsequente Grenzsicherung. Vgl. Arens u. Klein (2004), 494. 
gegen das Chanat als Vasall der Hohen Pforte ideologisch rechtfertigen. Der Erfolg blieb aber vorerst aus, denn sowohl 1687 als auch 1689 gelang es den tatarischen Truppen unter dem Kommando des Chans Selim I. Giray (1631-1704), eine dauerhafte Einnahme der Halbinsel und das russische Festsetzen an der nördlichen Schwarzmeerküste zu verhindern. Ein ausländischer Zeitgenosse führte diesen Misserfolg Golicyns weniger auf die Leistung der Krimtataren im Kampf, sondern auf deren Geschick am Verhandlungstisch zurück: Die krimtatarischen Unterhändler hätten die Gespräche mit dem Feldherren so in die Länge gezogen, dass der russischen Armee der Nachschub ausgegangen sei und sie sich deshalb habe zurückziehen müssen. Überhaupt seien die Feldzüge gegen das Chanat für die „Moskowiter“ ein Schlag ins Wasser gewesen. ${ }^{24}$

Erst unter Peter I. (1672-1725) sollte sich dies ändern - zumindest temporär gelang diesem gegenüber dem Osmanischen Reich ein Gebietsgewinn: die Festung von Azov, das ehemalige italienische Tana. Sowohl die Dnepr- (1559) als auch die Don-Kosaken (1637) hatten sich in der Zeit davor mit unterschiedlichem Erfolg an deren Eroberung versucht; letztere konnten die Feste zwar einnehmen, Zar Michail I. (1596-1645) gab sie jedoch bereits 1642 den Osmanen zurück, um kriegerische Auseinandersetzungen $\mathrm{zu}$ vermeiden. 1696 eroberte Peter I. schließlich Azov, nachdem ein erster Versuch ein Jahr vorher gescheitert war. ${ }^{25}$ Auch wenn er die Stadt einige Jahre später - nach dem Frieden vom Pruth, der den vierten „Russischen Türkenkrieg“ 1711 beendete - wieder zurückgeben musste, war das Ziel, Russland Zugang zum Schwarzen Meer zu verschaffen, deutlich zutage getreten. Das Krim-Chanat, welches eine Funktion als Pufferzone zwischen Russland und dem Osmanischen Reich hatte, musste zur Umsetzung dieses Plans ausgeschaltet werden, was mittelfristig gelingen sollte.

Die innenpolitische Lage des Chanats wurde parallel zu diesen internationalen Entwicklungen zunehmend instabiler. Ein Indikator dafür war die seit dem ausgehenden 17. Jahrhundert große Zahl an Herrscherwechseln, wobei eine ganze Reihe von Chanen mehrfach ein- bzw. abgesetzt wurde. ${ }^{26}$ Dies weist darauf hin, dass entweder die einflussreichen Mirza oder aber der osmanische Sultan mit dem jeweiligen Herrscher unzufrieden waren - und dieser sich wiederum nicht gegen die partikularen Interessen durchsetzen konnte. Unter den Clan-Führern gab es unterschiedliche Lager, nämlich solche, welche die osmanische Oberherrschaft grundsätzlich in Frage stellten und von den Chanen verlangten, das Verhältnis zur

24 Vgl. hierzu den aus dem Französischen übersetzten zeitgenössischen Bericht eines polnischen Gesandten am Moskauer Hof Zapiski (1891).

25 Dazu Fisher (1973).

26 Hammer-Purgstall (1970), 22, nennt insgesamt 54 verschiedene Chan-Herrschaften. Eine ganze Reihe von Girays übernahm mehrfach die Chanschaft. 
Hohen Pforte im Sinne einer größeren Unabhängigkeit neu zu regeln. Seit den 1760er Jahren waren separatistische Strömungen unter der Führung der Clane der Şirin und Barın dominierend, welche sogar die Unabhängigkeit vom Osmanischen Reich erreichen wollten. Daneben gab es aber diejenigen Mirza, die mit dem Sultan und dessen Macht, Chane ein- oder auch abzusetzen, durchaus zufrieden waren, war dies doch ein Hebel, um unliebsame, gegenüber den Mirza zu selbständig agierende Herrscher loszuwerden. ${ }^{27}$ Die Hohe Pforte ihrerseits forderte immer häufiger krimtatarische Truppen an, die u.a. im Kaukasus oder in Persien die Interessen Istanbuls durchsetzen sollten - und zwar zuweilen gegen den erklärten Willen der Clan-Führer. Abermals für Unruhe sorgten überdies „des KrimChans unsichere Kantonisten“28, die Nogaier, die wiederholt auch von den Clans für ihre Zwecke bei der Ein- oder Absetzung von Chanen instrumentalisiert wurden. ${ }^{29}$

Als Folge des Friedens von Karlowitz 1699 zwischen dem Osmanischen Reich auf der einen und dem Heiligen Römischen Reich, Polen-Litauen, Venedig, dem Kirchenstaat sowie Russland auf der anderen Seite kam es zu einer dieser Rebellionen der Nogaier, bei der sich der zweimalige Chan Devlet II. Giray (16481718; Chanschaften 1699-1702 bzw. 1709-1713) erstmalig als entschlossener Herrscher zeigte. ${ }^{30} 1701$ gelang ihm die Niederschlagung des Aufruhrs der Nogaier, die gegen das im Vertrag von 1699 festgesetzte Verbot des Eindringens und der Raubzüge in die nördlichen Anrainerstaaten des Chanats rebellierten. Ein zweites Mal machte Devlet II. Giray im Zuge des Großen Nordischen Krieges (1701-1721) von sich reden. Der grundsätzlich eher an der Sicherung der Position des Zarenreichs an der Ostsee und dem Baltikum interessierte Zar Peter I. musste sich im Verlauf dieses Waffenganges auch mit dem Chanat und Devlet II. Giray auseinandersetzen: Nach dem Sieg des Zaren bei Poltava über die Schweden und einen Teil der verbündeten Kosaken 1709, der den Wendepunkt in diesem Krieg bedeutete, betrieb Devlet II., nunmehr das zweite Mal Chan der Krim, eine Politik, die in Istanbul nicht auf Zustimmung traf ${ }^{31}$ : Auch um das Vordringen des Zarenreichs nach Süden zu verhindern, unterstützte er die mit den Schweden ver-

27 Vgl. Fisher (1977); Fisher (1978), 50.

28 So die Turkologin Barbara Kellner-Heinkele auf einer Tagung zur Geschichte des Krim-Chanats 2008 in München. Vgl. das Tagungsprogramm: Krimkhanat (2008).

29 Vgl. Hammer-Purgstall (1970), 194-198.

30 Zur Bewertung der Rebellion von 1699-1701 in der tatarischen und osmanischen Historiographie vgl. Klein D. (2012b). Zum Friedensschluss von 1699 vgl. Molnár (2013).

31 Ich folge hier vor allen Dingen Vozgrin (1992), 238-244. 
bündeten Kosakenverbände unter Ivan Mazepa (1639-1709) ${ }^{32}$ genauso wie die geschlagenen schwedischen Truppen unter ihrem König Karl XII. (1682-1718), denen er Zuflucht im Chanat gewährte. Zugleich warnte er die Russland gegenüber auf Ausgleich bedachte Hohe Pforte vor weiter reichenden russischen Ambitionen gegenüber dem Osmanischen Reich und dem Chanat. Um diesen entgegenzutreten, marschierte Devlet eigenständig 1711 mit einer Streitmacht von über 80.000 Tataren sowie Mazepa-Kosaken und schwedischen Soldaten Richtung Norden. Die Pforte, die aus Angst vor einem weiteren Krieg mit dem Zarenreich die Warnungen des Chans anfänglich ignoriert hatte, musste nolens volens abermals den Krieg mit Russland beginnen, der dann im Sommer desselben Jahres mit dem Frieden vom Pruth endete. Unter einem Vorwand - einem angeblich unangemessenen Auftreten gegenüber Karl XII. - ließ Sultan Ahmed III. (1673-1736) den eigenständig agierenden Devlet II. im Jahr 1713 abermals absetzen und nach Rhodos verbannen. Der russische Historiker Vozgrin sieht hier einen Wendepunkt in der Geschichte des Chanats: „Ab jetzt konnten die Chane nur warten, wer von den mächtigen Nachbarn als erster die Hand nach dieser reifen Frucht ausstrecken würde - die Türkei [sic!], Russland oder Polen. “33 Es war letztlich das Russländische Imperium, welches erfolgreich die Hand nach der Krim ausstreckte, jedoch dauerte es bis zum Vollzug noch einige Jahrzehnte.

Unter der Zarin Anna Ivanovna (1693-1740) rückten das Schwarze Meer sowie das Verhältnis zum Osmanischen Reich und damit auch zum Krim-Chanat wieder stärker in den Fokus St. Petersburgs, zumal die Verhältnisse im Norden durch den Sieg im Großen Nordischen Krieg im Sinne des Zarenreichs bzw. Peters I. geregelt worden waren. Der nächste Russisch-Osmanische Krieg von 1735 bis 1739 wurde allerdings insgesamt kein Erfolg für das Zarenreich, waren die territorialen Gewinne doch gering; immerhin ging Azov nun aber dauerhaft in russische Hände über. Gravierend waren die wirtschaftlichen Auswirkungen, denn Istanbul beschnitt die Handelsrechte russischer Händler im Schwarzen Meer in den folgenden Jahren. Immerhin gelang den russischen Militärs unter Burkhard Christoph Graf von Münnich (1683-1767) erstmalig der Vorstoß auf die Halbinsel sowie nach Bağçasaray und damit die Einnahme des Machtzentrums des langjährigen Gegners. Die Freude der russischen Emissäre dauerte aber nicht lange, denn abermals standen Nachschubprobleme ${ }^{34}$ und diesmal auch das Auftreten von Seuchen

32 Vozgrin (1992), u.a. 240f., antizipiert die Position der ukrainisch-nationalen Geschichtsschreibung, nach der die Zaporoger Kosaken eine protonationale Staatlichkeit repräsentierten und schreibt von einem krimtatarischen-ukrainischen Rapprochement.

33 Vozgrin (1992), $245 \mathrm{f}$.

34 Nach Hosking (1997), 48, hatten die Krimtataren „vorausblickend ihre Getreidespeicher angezündet und ihre Brunnen vergiftet.“ 
(vermutlich der Pest) einer dauerhaften Einnahme der Krim entgegen. ${ }^{35}$ Für die Wirtschaft und Infrastruktur des Chanats waren die Folgen des russischen Einmarsches gleichwohl bedeutend, hinterließen die Eindringlinge doch eine Spur der Zerstörung: „Münnich’s [sic!] Heereszug durch die schöne Krim verödete Felder und Städte“, so das Urteil Hammer-Purgstalls; aber auch zarische Historiker wie Vasilij Ključevskij kritisierten die Brutalität, mit der die russischen Militärs auf der Halbinsel vorgegangen waren. ${ }^{36}$ Durchaus als bewusste Symbolpolitik darf die teilweise Niederbrennung des Chan-Palasts durch die Invasoren gedeutet werden, ist die Zerstörung der kulturellen Insignien der gegnerischen Seite doch elementarer Teil kriegerischer Auseinandersetzungen. Bei dem Brand in der Hauptstadt des Chanats ging überdies ein Großteil des im Palast verwahrten krimtatarischen Schrifttums verloren, ${ }^{37}$ was Fachleute als einen unersetzlichen Verlust bezeichnen. ${ }^{38}$ Nach dem Rückzug der russischen Armee wurde der Palast zwar schnell in Teilen, aber nie mehr in seinen ursprünglichen Ausmaßen wiedererrichtet. $^{39}$ Der Niedergang des einst so machtvollen Chanats zeigte sich nunmehr auch materiell.

Auch wenn weder eine dauerhafte Einnahme noch die Vernichtung des KrimChanats zu diesem Zeitpunkt gelungen war, zeichnete sich doch die Machtverschiebung zugunsten des Russländischen Imperiums ab. Zugleich war eine neue ideologische Qualität sichtbar geworden: Die Sicherung der offenen, volatilen Grenzen und die Verhinderung des Verlusts von Menschen war lange Zeit die vordringliche und in Anbetracht der Lage auch dominierende Aufgabe des Moskauer Reiches bzw. des Zarenreichs gewesen; und da die Einfälle krimtatarischer Truppen auch noch bis in die zweite Hälfte des 18. Jahrhunderts andauerten - der letzte Raubzug fand 1769 unter Kurım Giray (1717-1769) statt -, ist dies nachvollziehbar. Das religiöse Motiv erfuhr, wie bereits erwähnt, in der zweiten Hälfte des 17. Jahrhunderts einen Bedeutungszuwachs, zumal sich das Moskauer Reich der antiosmanischen Koalition anschloss, die ihr Vorgehen eben auch als Kampf der Christenheit gegen den Islam verstand. Letztlich war - und dies wurde im Verlauf

35 Auf Seuchen und Quarantänemaßnahmen wird noch zurückzukommen sein. Vgl. auch Robarts (2017), besonders das Kapitel „Instruments of Despotism (II): Epidemic Disease, Quarantines, and Border Control in the Russian Empire“, 139-168.

36 Hammer-Purgstall (1970), 205; Kliutschewskij (1945), Bd. 2, 66; Seymour (1855), 28. Dieses im Kontext des Krimkriegs veröffentlichte Werk diente der Desavouierung Russlands.

37 Vgl. Jaremčuk u. Bezverchyj (1994), 21, wo das 1833 auf Geheiß der Verwaltung erfolgte Einsammeln und Verbrennen krimtatarischer Bücher und Urkunden genannt wird.

38 Vgl. dazu die bei Fisher (1978), 213, Anm. 3, angegebene Literatur.

39 Nach Fisher (1978), 51, war der teilweise Wiederaufbau des Palasts nach dem Abzug der russischen Truppen innerhalb von nur drei Jahren eine beeindruckende Leistung. 
des 19. Jahrhunderts mit der Kulmination im Krimkrieg von 1853 bis 1856 noch deutlicher ${ }^{40}$ - die Krim-Frage aber deutlich komplexer. Sie wird von der Forschung als Teil der so ausführlich behandelten sog. Orientalischen Frage interpretiert. ${ }^{41}$ Daran zeigt sich, dass eine Reduktion dieses Themas auf die religiöse Differenz unzulässig ist.

Die Konkurrenz der europäischen Großmächte um den Einfluss auf das Osmanischen Reich hatte im Verlauf des 18. Jahrhunderts ihren Anfang genommen und wird zumeist mit entstehenden technologischen, militärischen und ökonomischen Defiziten der einst so mächtigen Hohen Pforte erklärt. Aus diesen versuchten die europäischen Großmächte ihren Nutzen zu ziehen. Nationale Bewegungen innerhalb des Osmanischen Reiches wie die der Griechen, auf die auch der Dichter Binhack angespielt hatte, wurden von diesen genauso instrumentalisiert wie die religiöse Differenz zwischen Islam und Christentum. Der russischosmanische Gegensatz war also ein wichtiges, allerdings nicht das einzige Element bei der Entstehung der Orientalischen Frage. Auch Frankreich - im 18. Jahrhundert zumeist ein wesentlicher Unterstützer der Hohen Pforte -, welches sich nach der Revolution von 1789 und der ägyptischen Expedition Napoleon Bonapartes 1798/99 aber auf Kosten Istanbuls zu bereichern suchte, Großbritannien oder das Habsburgerreich taten Einiges, um die sog. Orientalische Frage zu einem Dauerbrenner der europäischen Diplomatie zu machen. Die sog. Meerengenfrage schließlich - und damit die Schwarzmeerregion - war ein wesentliches Problem in diesem Kontext. ${ }^{42}$

Welche Rolle spielte die Halbinsel in dieser internationalen Gemengelage? Offenbar keine geringe: Für den Verfasser einer Geschichte des Schwarzen Meeres, Charles King, ist die Krim-Frage regional und substantiell deren „true heart“ gewesen; mit dieser seien die Meerengenfrage, aber eben auch und vor allen Dingen der Krimkrieg und dessen Folgen verbunden. ${ }^{43}$ Ähnlich sah es der deutsche Historiker Edgar Hösch bereits vor mehr als fünfzig Jahren in einem immer

40 Dazu immer noch Baumgart (1972). Vgl. auch Figes (2010), in der deutschen Übersetzung mit einem programmatischen Untertitel: „Der Krimkrieg. Der letzte Kreuzzug“, Figes (2011).

41 Ich beziehe mich hier im Wesentlichen auf die ältere, aber immer noch relevante Arbeit Anderson M. (1966), sowie Bitis (2007) und Frary u. Kozelsky (2014). Vgl. dort auch die Einleitung (3-34) mit einem aktuellen Forschungsüberblick, der allerdings die deutschsprachigen Beiträge nicht berücksichtigt.

42 Vor allen Dingen Großbritannien und die Habsburgermonarchie bevorzugten lange Zeit ein Mächtegleichgewicht und wollten die territoriale Integrität des Osmanischen Reiches zumindest de jure aufrechterhalten; gegen eine starke wirtschaftliche und politische Einflussnahme auf das Osmanische Reich hatten allerdings alle Großmächte nichts einzuwenden. Nach dem Krimkrieg wurde die Orientalische Frage mehr und mehr zur Balkanfrage.

43 King (2004), 5. 
noch maßgeblichen Aufsatz, in dem er Folgendes festhielt: Für die russische Diplomatie habe die Orientalische Frage lange Zeit ausschließlich im Verhältnis zum Krim-Chanat gelegen, ${ }^{44}$ was in Anbetracht der ausführlich geschilderten Bedrückungen, die für die ost- (und west-)slavischen Gebiete lange Zeit vom Krim-Staat ausgingen, auf der Hand lag. Nun waren zwar bis 1769 immer noch Einfälle krimtatarischer Reiter zu beklagen, deren Ausbeute war aber aufgrund besserer Abwehrmaßnahmen und einer erfolgreichen russischen Offensivstrategie zunehmend geringer geworden. Alsbald kamen jedoch russischerseits weitere Motive hinzu, welche auf die Ausschaltung des Pufferstaates Krim-Chanat zielten: Die von osmanischer Seite wiederholt aufgebauten Handelserschwernisse für russische Kaufleute (s.o.) verärgerten St. Petersburg. Wiederholt beschwerten sich die Zaren bei der Hohen Pforte darüber, und besonders nachdrücklich tat dies Katharina II.: „In ihren wiederholten Beschwerden über die Pforte hat sie [Katharina II.] eine Förderung und Sicherung der freien Handelsschiffahrt auf dem Schwarzen Meer mit besonderem Nachdruck betrieben und ihr wachsendes Interesse an der wirtschaftlichen Erschließung des Südens bekundet“45, so die Feststellung Höschs. Sie war aber nicht die erste gewesen, denn schon gegen Ende der Regierungszeit Elisabeth Petrovnas (1741-1762) hatten Privatpersonen im Zarenreich ein verstärktes Interesse am Schwarzmeerhandel formuliert. Ein Resultat war 1759 die Beauftragung einer Studie über neue Handelswege zwischen dem Het'manat und Istanbul durch Handelskammer und Senat. Diese Routen würden in jedem Fall durch das Chanat führen und schon deshalb besondere militärische, und damit auch staatliche, Schutzmaßnahmen erfordern, so hieß es in der Studie. Ein 1762 und damit nur wenige Monate nach dem Regierungsantritt Katharinas II. verfasster Bericht des Hofrats Dmitrij Ladygin über die wirtschaftlichen Aussichten des Schwarzmeerhandels weckte das Interesse der neuen Zarin. Die Perspektive, zukünftig eine größere Rolle im profitablen Schwarzmeerhandel spielen zu können, dürfte den Beginn des für die Geschichte der Krim so folgenreichen Russisch-Osmanischen Kriegs von 1768-1774, auf den noch zurückzukommen ist, in jedem Fall begünstigt haben. ${ }^{46}$

Als Teilaspekt der sog. Orientalischen Frage im 18. Jahrhundert wird das „Griechische Projekt“ angesehen; dieses sei, so noch einmal Hösch, der Versuch Russlands gewesen, jenes Problem zu lösen. ${ }^{47}$ Seit Herrschaftsantritts Katharinas II. 1762 konkretisierte sich der Plan zur Aufteilung des europäischen Teils des Osmanischen Reichs zwischen Russland, das die nördliche Schwarzmeerküste

44 Hösch (1964), 196.

45 Hösch (1964), 196.

46 Hierzu und zum Bericht Ladygins vgl. Jones (1996), $125 \mathrm{f}$.

47 Hösch (1964), 202. 
gewinnen wollte, den Habsburgern, die Interessen an den Donaufürstentümern Moldau und Walachei formulierten, und der Republik Venedig. Die Region um Car'grad, wie Istanbul in der russischen Diktion hieß, sollte nach diesem „Griechischen Plan“ eine Sekundogenitur der Romanovs werden. Die Forschung bringt dies zuweilen mit der Taufe des zweitgeborenen Enkels Katharinas auf den Namen Konstantin im Jahr 1779 in Verbindung; dieser sei überdies von „eigens nach Moskau geholten griechischen Ammen“ gesäugt worden und mit griechischen Spielgefährten aufgewachsen, was manchen als Beweis gilt, dass Konstantin für diese Aufgabe auserwählt worden sei. ${ }^{48}$ An dieser Stelle kann nicht überprüft werden, ob die Erziehung Konstantins ursächlich mit den Aspirationen Russlands gegenüber dem Osmanischen Reich verknüpft war. Allerdings ist unstrittig, dass ein sog. Griechischer Plan schon älter ist: Es gibt Hinweise, dass eine Art Griechisches Projekt, zu dessen Verwirklichung die Beseitigung des Krim-Chanats ja eine Voraussetzung war, schon während der Herrschaft Alexej Michajlovičs und der Zarin Anna Ivanovna konturiert wurde (s.o.).

Im Verlauf der 1770er Jahre wurden die russischen Planungen zur Eliminierung des strategisch relevanten Krim-Chanats konkret; über die Form wurde am Zarenhof aber kontrovers debattiert: Der bis 1781 die russische Außenpolitik gestaltende Nikita I. Panin (1718-1783) ${ }^{49}$ plädierte für eine indirekte politisch-militärische Einflussnahme auf das Krim-Chanat. Kanzler Michail I. Voroncov (17141767) und bald auch andere sprachen sich indes für die direkte russische Herrschaft über die Krim aus. Voroncov übergab Katharina II. nach ihrer Thronbesteigung ein Memorandum mit dem bezeichnenden Titel „Bericht an Kaiserin Katharina II. nach Ihrer Thronbesteigung, das System der Krimtataren schildernd, deren Gefährlichkeit für Russland und die Forderungen an sie. Über das Kleine Tatarien.“ Darin wurden das Chanat im Kontext des russisch-osmanischen Verhältnisses behandelt und dessen tatarische BewohnerInnen in eingeführter Diktion als gefährlich und wesentliches Hindernis zur Erlangung der Dominanz des Zarenreichs über das Schwarze Meer dargestellt. ${ }^{50}$ Solange die Krim ,in türkischer Untertänigkeit bleibt, wird es für Russland immer gefährlich sein“, hieß es in dem Memoire Voroncovs - und überhaupt befände sich im Süden alles, u. a. wegen der

48 Hösch (1964), $183 \mathrm{f}$.

49 Panin sah - in einer älteren Tradition stehend - Russland als eine, nordische‘ Macht an; dies war eine Meinung, die bis zum Ende des Krimkriegs innerhalb Russlands häufig anzutreffen war. Die imperialen Ambitionen im Süden stießen deshalb bei ihm eher auf Ablehnung. Zum Wandel der russischen kollektiven Eigenverortung von einer ,nordischen` zu einer, östlichen Macht‘ vgl. Lemberg (1985).

50 Voroncov (1916). 
eigenmächtigen Kosaken, in Unordnung und bedürfe der Herstellung von Ordnung durch die russische Macht. ${ }^{51}$

Den von Vasilij M. Dolgorukov (1722-1782) befehligten zarischen Truppen gelang 1771 im Verlauf des fünften Russisch-Osmanischen Krieges die Einnahme großer Teile der Krim. Doch anders als bei der ersten Besetzung unter von Münnich einige Jahrzehnte vorher agierte man dieses Mal gegenüber der Zivilbevölkerung mit größerer Vorsicht. ${ }^{52}$ Katharina II. machte sich sogar die - am Hof von St. Petersburg offenbar bekannten - Dispute unter den Clans über das Verhältnis zwischen dem Chanat und Istanbul zu Nutze: Sie ließ ein Manifest veröffentlichen, in dem den Krimtataren die Wiedererlangung der verlorenen Unabhängigkeit versprochen wurde. Diese seien unschuldige Sklaven der Pforte gewesen, aber nun durch Russland befreit worden. ${ }^{53}$ Die Machtverhältnisse hatten sich zwar umgekehrt, von einer regelrechten Inkorporation des Chanats in das Zarenreich wollte Katharina, die somit dem ,Modell Panin“ und nicht dem ,Modell Voroncov' folgte, dennoch nichts wissen - noch nicht.

In dem im Juli 1774 zwischen St. Petersburg und der Hohen Pforte geschlossenen Friedensvertrag von Küçük Kaynarca (türk.; russ. Kjučuk-Kajnardža) sicherte sich das Zarenreich u.a. die Dnepr-Mündung (und damit Zugang zum Schwarzen Meer) sowie Teile des Nordkaukasus und durfte in den bislang von der Pforte kontrollierten Gewässern ohne Einschränkung Handel betreiben. Die bisher von den Osmanen gehaltenen Festungen Kerč’, Yeñi Qale (krimtat.; russ. Enikale; ukr. Jenikale) und Kinburn (russ./ukr.; türk. Kılburun) gingen in russische Hände über. ${ }^{54}$ Das Krim-Chanat wurde zwar von Russland als auch dem Osmanischen Reich als ein unabhängiger Staat bezeichnet, es stand aber tatsächlich unter starkem russischen Einfluss. Allerdings behielt der Sultan in Rückbesinnung auf seine Kalifen-Würde in religiösen Angelegenheiten Mitsprache im Krim-Staat, so dass der Vertrag von 1774 weder eine völlige Niederlage der Pforte noch ein totaler russischer Triumph war..$^{55}$

Für das seit 1478 eng mit dem Osmanischen Reich verbundene Krim-Chanat begann 1774 eine neue und (wie sich zeigen sollte) nur kurze Phase der formalen Unabhängigkeit.

51 Voroncov (1916), $191 \mathrm{f}$.

52 Fisher (1970), 41.

53 Fisher (1978), 54. Vgl. den vollständigen Text: Forma manifesta (1896).

54 Hierzu immer noch grundlegend Družinina (1955). Nach Anderson M. (1966), XI, gilt dieser als einer der wichtigsten Friedensschlüsse der europäischen Diplomatiegeschichte überhaupt. 55 Vgl. die unterschiedlichen Positionen bei Davison (1976) bzw. Weisband (1973), 211. 